\title{
A Control Plane Architecture for Multi-Domain Elastic Optical Networks: the view of the IDEALIST project
}

\author{
Ramon Casellas (1), Oscar González (2), Francesco Paolucci (3), Roberto Morro (4), Víctor López (2),
} Daniel King (5), Ricardo Martínez (1), Filippo Cugini (3), Raül Muñoz (1), Adrian Farrel (5), Ricard Vilalta (1), Juan-Pedro Fernández-Palacios (2)

(1) CTTC, Spain

(2) Telefónica Research and Development (I+D), Spain

(3) CNIT-Scuola Superiore Sant'Anna, Italy

(4) Telecom Italia, Italy

(5) Old Dog Consulting, UK

\begin{abstract}
A key objective of the IDEALIST project included the design and implementation of a Generalized MultiProtocol Label Switching (GMPLS) and Path Computation Element (PCE)-based control plane for multivendor and multi-domain flexi-grid elastic optical networks (EON), leveraging the project advances in the optical switching and transmission technology, an enabling interoperable deployment. A control plane, relying on a set of entities, interfaces and protocols, provides the automation of the provisioning, recovery and monitoring of end-to-end optical connections. This paper provides an overview of the implemented architecture; we present the macroscopic system along with the core functional blocks, control procedures, message flows and protocol extensions.
\end{abstract}

The implemented end-to-end architecture adopted active stateful hierarchical PCE, under the control and orchestration of an adaptive network manager, interacting with a parent PCE that first, coordinates the selection of domains and the end-to-end provisioning using an abstracted view of the topology and, second, delegates the actual computation and intra-domain provisioning to the corresponding children PCEs. Endto-end connectivity is obtained by either a single Label Switched Path (LSP), or by the concatenation of multiple LSP segments, which are set up independently by the underlying GMPLS control plane at each domain.

The architecture and protocol extensions have been implemented by several partners, assessing interoperability in a multi-partner testbed and adoption by the relevant Internet standards development organisations (SDO).

\section{Introduction}

\section{a. Flexi-grid Networks}

Optical Transport Networks [1] (OTN) are composed of network elements connected by optical fibers allowing the transport, multiplexing, routing, management, supervision and survivability of optical channels carrying client signals. Such channels were constrained by a DWDM fixed frequency grid, inefficient for low rate signals and not adequate for high rate ones. The term "flexible grid or flexi-grid" [2] relates to the updated set of Nominal Central Frequencies (NCF) -- defined within an abstract grid anchored at $193.1 \mathrm{THz}--$, a new channel spacing $(6.25 \mathrm{GHz})$ and other optical spectrum management considerations covering the efficient and flexible allocation of optical spectral bandwidth. A frequency slot (i.e., a variable-sized optical frequency range) is thus characterized by its nominal central frequency and its width, expressed in multiples of a given width granularity $(12.5 \mathrm{GHz})$, and can be allocated to a connection, based on the signal modulation format and data rate.

The functional architecture of an OTN is decomposed into independent layers [1] and, in our context, the media layer is the server layer of the optical signal layer and the optical signal is guided to its destination by means of a network media channel where the switching is based on a frequency slot.

\section{b. Hardware Models}

An information model is an abstract description used to represent and manage objects (such as a network device) a conceptual level, independent of any specific protocols used to transport data. A data model is 
protocol specific and includes many of technology specific details. Using well-defined standards-based common information and data models, provides interoperable data exchange between different implementations.

Standardisation, notably at the Internet Engineering Task Force (IETF), is often influenced by early implementations and cooperative development by vendors and open source projects. Particularly pertinent to this paper is the fact that the data models that were used to represent and configure optical interfaces with flexi-grid capabilities, or to describe a network topology (nodes, links, and connectivity) enhanced with details of optical capabilities and available resources, enabling network optimization and dynamic and online path computation, were developed by the project members themselves and contributed to the IETF.

\section{c. Drivers and Motivations for an "IDEALIST" Control Plane}

Backbone networks are intended to transport the aggregated traffic from several metropolitan networks. However, existing transport networks are based on the assumption that the traffic demands are predictable, and are not adapted to varying traffic requirements. Therefore, current networks require multiple manual configurations in the metro and core network nodes.

Dynamic optical networks are possible thanks to a distributed Generalized Multi-Protocol Label Switching (GMPLS) control plane. There is a need of an end-to-end architecture to reduce the provisioning process of legacy Network Management Systems (NMS), using standard network configuration interfaces, which will trigger automated standard control plane for multi-domain/vendor/layer operation. The control plane allows the reconfiguration of the optical service, its protection and restoration capabilities, not only for a single domain, but also for multi-domain scenarios. The benefits of a standardized control plane extend beyond the absolute functions enabled by the control plane itself, because such a common approach also facilitates interoperability between equipment supplied by different vendors and so enables a network operator to construct a heterogeneous network yet operate it in a homogeneous way.

The implemented control plane architecture covers the automated provisioning and recovery of network connectivity services in a multi-domain setting. Such developments are increasingly driven by use cases such as interconnecting distributed data-centers, associated traffic patterns and dynamicity.

\section{d. Existing Control Plane Framework}

There is extensive experience in the use of a dynamic distributed control plane. Standardization of this work has been conducted principally within the IETF, with some architectural and use-case documents developed within the ITU-T. The GMPLS architecture [3] comprises:

- A link/neighbor discovery/verification protocol, such as the Link Management Protocol (LMP), that allows neighboring nodes part of a he control plane adjacency to unambiguously associate data plane adjacencies (e.g. fiber links), correlate identifiers and to assure compatible capabilities.

- $\quad$ A routing protocol. The Open Shortest Path First (OSPF) protocol describes the characteristics of nodes and links, so the state and capabilities of the resources are distributed and updated to all of the nodes, knowing which resources are in use, faulted/out of service, or available.

- $\quad$ A signaling protocol. The ReSerVation Protocol with Traffic Engineering extensions (RSVPTE) is used to set up Label Switched Paths (LSPs). RSVP-TE messages specify the path of the LSP, request specific capacity on the path, and report back the exact allocated network resources to support the LSP.

- A path computation service. A key aspect is determining what path an LSP should follow. This function can be performed externally (the path is supplied to the control plane), or delegated to the control plane. In either case, the computation can be complex. The Path Computation Element $(\mathrm{PCE})$ is a functional component that can be queried using the Path Computation Element communication Protocol (PCEP), recently extended to allow the network to delegate control of an LSP to a PCE, and to allow a PCE to direct the establishment of new LSPs (becoming an active PCE) [4].

A network state reporting mechanism. Link State Border Gateway Protocol (BGP-LS) allows an entity to collect, synthesize, and report the full set of state and capability information from the network to an external consumer such as a management system [5]. 

by the IETF through their Application Based Network Operation (ABNO) architecture [6].

\section{II. Control Plane Architecture}

4 Our GMPLS/PCE control plane for multi-domain flexi-grid networks addresses the provisioning of either a network media channel or a constant bit rate service between optical transceivers, which can support multiple bit rates. A media channel is a media association representing the topology path and the allocated resource (i.e., the frequency slot). It is similar to the GMPLS concept of LSP where, from a data plane perspective, it is the path in the network resulting of reserving and configuring transmission and switching resources across TE links and nodes in a way that in can transport client signals and data from its entry point or interface to the exit point or interface. It represents a (effective) frequency slot supported by a concatenation of media elements. GMPLS labels locally represent the media channel and its associated frequency slot, which is the switched resource. Network media channels are considered a particular case of media channels when the end points are transceivers, and transport a single Optical Tributary Signal (OTS), as shown in Figure 1. The control plane deals with the resource reservation and configuration of media layer matrixes that switch frequency slots and the configuration of the transceivers at the endpoints, with an agreed hardware model that, as of today, is not standard. No signal layer (e.g. OTS) switching is considered. Switching at the media layer is configured by configuring optical filters and configuring cross-connections.

From a bottom-top approach, each domain deploys its own GMPLS control plane instance. On top of it, each domain deploys an active stateful PCE (AS-PCE) for the purposes of both optimal path computation and service provisioning within its domain. Multi-domain path computation and provisioning is carried out by means of a Hierarchical Path Computation Element (H-PCE) [7], with the parent PCE (pPCE), coordinating the procedures between children PCEs (CPCE) and under the control and orchestration of an Adaptive Network Manager (ANM). The macroscopic architecture is shown in Figure 2.

\section{a. Adaptive Network Manager and In-Operation Network Planning}

The control plane has relied only on distributed functionalities, but the advent of PCE demonstrates that having a central entity can provide multiple benefits. The ANM was conceived with the idea of orchestrating network processes beyond the PCE capabilities. Its functionalities are to monitor network resources, and to decide the optimal network configuration based on the status, bandwidth availability and user service. It does not replace the control plane, but extends and complements it (e.g. interacting with the client layer) and delegating specific functions (e.g. path computation) to it.

The ANM was implemented, utilising the ABNO architecture, and relies on standards-based and open interfaces, providing the capability for application interaction via a North Bound Interface (NBI) and South Bound Interface to the data plane, either directly to each network element or via the control plane. The link between the ANM and the control plane is the parent PCE, which receives queries to carry out path computation and provisioning end-to-end connections.

The ANM platform allows automatic IP Link provisioning, multi-layer restoration, dynamic bandwidth allocation based on traffic changes, periodic defragmentation and network reoptimization after network failure recovery [8], so an operator planning tool has updated network information and maintains a provisioning interface with the network. This architecture benefits from the GMPLS/PCE control plane, reducing network CAPEX by minimising the over-provisioning required in today's static environments.

\section{b. Hierarchical Path Computation Element}

A parent PCE (pPCE) is responsible for inter-domain path computation, while in each domain a local child PCE (cPCE) performs intra-domain computation. The pPCE resorts to the Hierarchical Traffic Engineering Database (H-TED) storing the list of the domains and inter-domain connectivity information, to determine the sequence of domains. Moreover, to perform effective inter-domain computation the pPCE is allowed to ask cPCEs for the path computation of the several border-to-border LSP segments.

A number of innovative extensions have been implemented by IDEALIST. First, besides reachability information, abstract intra-domain TE information is announced to the pPCE (e.g., in the form of mesh of abstracted TE links between border nodes) with the aim of improving the effectiveness of the domain sequence computation. In particular, the north-bound distribution of Link State and TE information using BGP-LS is utilized by domains' BGP speakers to populate the H-TED. Second, in order to enable advanced 
TE functionalities, e.g., elastic operations and re-optimizations [9][10], the H-PCE architecture has been extended to support the active stateful PCE with instantiation capabilities.

In summary, the H-PCE achieves end-to-end path computation by performing domain sequence selection and segment expansion, based on spectrum availability information provided by BGP-LS and PCEP requests submitted to CPCEs. The same H-PCE deployment is used in some use cases to perform the provisioning, where the end-to-end path is split in segments, sent to CPCE by means of instantiation messages and each CPCE performs segment instantiation: the end-to-end LSP is setup in the form of a "stitching on the wire" of several segments.

\section{c. GMPLS Distributed Control Plane}

Within each domain, there is an instance of a GMPLS control plane. GMPLS controllers execute several collaborative processes and a data communication network based on IP control channels allows the exchange of control messages between controllers. Noteworthy processes are the Connection Controller, the Routing Controller or the Link Resource Manager. We assume that a GMPLS controller is associated to a single flexi-grid optical node.

Under distributed control, each GMPLS controller manages the state of the connections (i.e., LSPS) originating, terminating or passing-through a node and maintains its own network state information (topology and resources), collected in a local TED and synchronized thanks to the routing and topology dissemination protocol. Controllers then appropriately configure the underlying hardware (filter, transceiver or switch configuration) during the establishment of an LSP, as per the basic operation of a GMPLS control plane [3]. In the next section, we overview the main involved procedures focusing on the specific aspects of the optical technology (see [11] for a detailed view).

\section{Control Plane Procedures}

\section{a. Intra-domain and Inter-domain Topology Dissemination}

Within a domain, each node routing controller is responsible for disseminating changes in the network state regarding the resources under its control (e.g., originating links) through OSPF-TE Link State Advertisements (LSA). Each LSA is sent to the neighboring nodes, which update their TED repositories and forward the LSA in turn. This mechanism allows synchronizing all the nodes' repositories within a given time, referred to as the routing convergence time. The basic procedures remain mostly unchanged, relying on extending the actual information objects within the LSAs.

OSPF-TE has been extended to support the dissemination of per-node and per-link TE attributes, reflecting device restrictions and overall optical spectrum availability. In particular, nodes may have asymmetric switching capabilities or different minimum slots size restrictions; optical transmitters/receivers may have different tunability constraints ... Other extensions have been implemented for disseminating the capabilities of Sliceable Bandwidth Variable Transceivers (S-BVTs), including, e.g., the number of available subtransponders and their parameters. Let us note that, in the approach, OSPF-TE is one of the methods by which a CPCE obtains the TED to perform constrained Routing and Spectrum Assignment (RSA) and is the source of the (abstracted) information conveyed towards the pPCE.

BGP-LS has been also suitably extended to support specific information exchange, such as spectrum availability, transponders' physical parameters and interoperability capabilities. BGP-LS is also used to report the relevant attributes of inter-domain links. Without disclosing the internal domain topology, this allows a pPCE having, at least, a graph that represents inter-domain connectivity and to perform basic multidomain path computation.

\section{b. Multi-domain Path Computation}

Following Figure 3, when a service request, driven by an operator, is received by the ANM, the controller asks the pPCE for an inter-domain path (step 1). The pPCE, based on the (possibly abstracted and aggregated) information obtained from the cPCEs computes the domain sequence (including each domain entry and exit nodes) and subsequently requests the CPCEs the corresponding border-to-border expansion (also by means of PCEP PCReq messages, step 2). Once the pPCE receives the responses (PCRep, step 3 ), which include, among other objects, the segment spectrum availability, the pPCE performs a detailed end-to-end path computation including the routing, spectrum assignment and transponder selection. Optical 
constraints are considered based, for instance, on nodes switching capabilities, optical reach, and transponder capabilities. For example, in the case of end-to-end spectrum continuity constraint, the pPCE has to assign a frequency slot such that i) it is able to convey the requested bandwidth, ii) it is available across all the end-to-end path, including inter-domain links, iii) allows the selection of available end-point transponders. Then, the PPCE answers the ABNO controller via a PCEP Response message.

\section{c. Inter-domain Service Provisioning via ANM with Active Stateful Capabilities}

Once the path is computed, the ABNO controller requests the pPCE to establish the path with a PCEP Initiate message. There are several provisioning models, with varying requirements of control plane interoperability. Here, we focus in i) the contiguous LSP with a single end-to-end RSVP-TE session and ii) the model relying on the stateful capabilities of the H-PCE structure with multiple (one per domain) RSVPTE sessions.

In the single session case, the provisioning interface is a dedicated PCEP session with either the cPCE of the ingress domain or directly the ingress node and there is a single RSVP-TE session from the source node within the source domain to the destination node. The multiple session case requires that all PCEs are stateful with instantiation capabilities. The connectivity at the data plane level is insured by concatenating compatible media channels at every domain, each set up by the local RSVP-TE session. Note that the first case implies interoperability at the control plane signaling level between different optical vendors' respective RSVP-TE implementations at the inter-domain boundaries, since there is a single end-to-end session that crosses the external network-to-network interfaces. On the contrary, for the second case, interoperability requirements are limited to PCEP, vertically, from the CPCEs to the pPCE, between each vendor and the provider of the pPCE. Both approaches can be seen in Figure 3 and Figure 4.

In either case, once the end-to-end path or the specific segment is computed, the assigned slot is included in the Explicit Route Objects (EROs) after each hop. In the first case (Figure 3 ) the end-to-end ERO is sent to the ingress node in a PCInitiate message (step 5), triggering the signaling process $(6,7)$ and final report to the ANM (8). In the second case (Figure 4), the obtained ERO per segment are enclosed in PCInitiate messages sent by pPCE to each involved CPCE (step 4). Once intra-domain provisioning is performed (step 5-8), PCE Report (PCRpt) messages are sent to pPCE to acknowledge the segments' status (step 9). Finally, the multi-domain LSP is stored in the H-TED and provisioning response is provided to the ANM (step 10). Similar procedures for inter-domain LSP update and LSP deletion are envisioned.

\section{Control Plane Protocol Extensions}

Control plane extensions affect all the protocols of the GMPLS suite together with the ones adopted as northbound interfaces (i.e. PCEP and BGP-LS).

\section{a. Provisioning and LSPDB Synchronization Interface}

The provisioning of LSPs relies on the use of the PCEP protocol, enhanced with stateful and instantiation extensions. Specific extensions to PCEP to cope with flexi-grid involve i) the BANDWIDTH object to convey the traffic descriptor that specifies the requested or allocated frequency slot width and ii) the ERO object with the resources to use along the path, which has been extended to carry the information describing the configuration of the optical transponders, such as the selected modulation format, baud rate, FEC, etc. To this end, a new sub-object, called Explicit Transponder Control (ETC) has been defined. It is formed by a variable list of Sub-Transponder TLVs, each of them describing one of the specific sub-carrier forming the super-channel LSP. To overcome scalability limitations, we enable the summarization of a set of parameters in a single parameter, the transceiver class, which considers the main parameters such as trunk mode and type, framing, channel band and grid, minimum and maximum chromatic dispersion, maximum polarization mode dispersion and differential group delay etc. A transceiver vendor is thus responsible for specifying the class contents and values. The vendor can publish the parameters of its classes or declare to be compatible with already published classes.

\section{b. Intra-domain Topology Dissemination}

The OSPF-TE protocol has been extended to convey, on a per link basis, the status of each possible central frequency or NCF (referred to as NCF availability) and the presence and attributes of transceivers. The former is done by means of a new object within the Switching Capability-Specific Information (SCSI) field. NCF availability is advertised using a bitmap format with bit position zero represents the lowest central 
frequency, each succeeding bit position represents the next central frequency and a bit set to 1 means the NCF is not in use.

\section{c. Multi-domain Topology Abstraction}

BGP-LS extensions addressed both the propagation of NCFs availability and the announcement of an SBVT transceiver capabilities to the pPCE, in order to perform Routing and Spectrum Assignment (RSA) for the multi-domain path. The first extension involves adding a new LINK_STATE attributes object TLV into the BGP-LS Update message, further characterizing a given optical link. The latter extension involves announcing the capabilities of an S-BVT attached to a given link using two new BGP-LS TLVs called "MFOTP encoding" (for multi-flow optical transponder) and "Transceiver class and application code" respectively. Both BGP-LS extensions reuse the same encoding as the ones proposed in OSPF-TE.

\section{d. Path Computation}

Specific extensions were defined for the RSA procedures in a hierarchical framework. Upon request from the pPCE, all cPCEs compute the path segment (sequence of nodes and links) inside their respective domain and reply this information to the PPCE, along with spectrum availability. This is accomplished by sending a PCEP Reply (PCRep) message containing the ERO object and two new objects: i) a LABEL_SET object that encodes the free NCFs along the computed path and ii) a SUGGESTED_LABEL object, suggesting (but not mandating) the label (i.e. the specific frequency slot) to be used in that domain. The pPCE performs an end-to-end allocation with this information.

\section{e. Signaling Protocol}

The extensions to the signaling protocol included: i) a new 64-bits label format, used in all the objects carrying a label (GENERALIZED_LABEL, SUGGESTED_LABEL, LABEL_SET, ERO, etc.) specifying frequency slot center and width in terms of two integer values, $n$ and $m$, according to the following formulas: Center Frequency $(\mathrm{THz})=193.1+\mathrm{n}{ }^{*} 0.00625$, Slot Width $(\mathrm{GHz})=12.5^{*} \mathrm{~m}$; ii) a new traffic descriptor type for the SENDER_TSPEC and FLOWSPEC objects to specify traffic parameters, carrying the slot width. Note that the label value, used in GMPLS to define what is switched, indicates, in this case, the slot features and, in particular, its width, therefore affecting also the LSP bandwidth. The same ERO extensions already described apply to the ERO object contained in the signaling messages.

\section{Experimental Validation}

The architecture and its integration with the underlying data plane has been demonstrated in several stages, starting from control plane testbeds and ultimately integrating both control and data planes. In [12] the optical channel provisioning was evaluated in a distributed multi-partner control plane testbed with locations in Madrid (Telefónica I+D), Barcelona (CTTC), Torino (Telecom Italia) and Pisa (CNIT). The testbed connected at the control plane level by means of dedicated IPsec tunnels, emulating a multi-domain network (Figure 5). On top of this connectivity, logical relationships between PCEs were established. We reported the details of the interoperability of routing (BGP-LS), path computation and instantiation (PCEP) and signaling (RSVPTE) implementations [12]. In [13], a higher degree of interoperability was achieved demonstrating the aforementioned different provisioning models. Experimental results showed all protocol interactions and LSP setup times. The adoption of BGP-LS extensions fully enabled multi-domain TE and was demonstrated in a limited number of domains. The system was integrated and demonstrated at both the control and data plane levels [14], where domains can have real hardware optical nodes that switch frequency slots although, by necessity, inter-domain links between remote locations are emulated. The data plane included both real and emulated flexi-grid nodes and SBVTs. Two real S-BVT prototypes were provided by different IDEALIST Vendors (e.g., CNIT/Ericsson and Coriant). These S-BVTs performed super-channel transmission with configurable number of PM-16QAM Nyquist-shaped carriers, overall providing up to 1Tb/s. At the receiver, coherent strategy with off-line post-processing was adopted. The S-BVTs supported the configuration of the number of active carriers, their central frequencies, modulation format, symbol rate and FEC.

\section{$47 \quad$ VI. Future Considerations}

The evolution of transmission and data plane technologies, supporting rates at $1 \mathrm{~Tb} / \mathrm{s}$ and beyond, will reach its maximum potential when supported by automatic configuration procedures enabling the deployment of spectrally-efficient plug-and-play transponders. Control plane solutions will have to be improved to provide procedures for commissioning and self-tuning of the transmission parameters (e.g., upon failure recovery) 

operate in interoperable multi-vendor environments.

While the control plane supports the dynamic configuration of transceivers, the full automation and selftuning of parameters will rely on the integration with functional components related to cognitive and selfadaptive networks. The solutions require, e.g., the deployment of passive and active monitoring and measurement systems beyond what currently exists, along with the adoption of formal languages and frameworks for the specification on rules and policies typical of expert systems.

The multi-vendor interoperability still remains a major issue to solve. While there are incentives (e.g. from operators or service providers trying to drive down costs), there is a huge pressure for vendors to increase margins and differentiate from competing offers.

The decoupling of data and control plane is expected to also be applied in the context of optical core networks through the concept of Transport SDN. A unified control plane architecture is expected to successfully orchestrate core with metro and data center premises, enabling the challenging support for future front/back-haul networks and $5 \mathrm{G}$ applications. Once flexible and open frameworks and interfaces have been adopted for the control and orchestration of network connectivity services across e.g. multiple heterogeneous domains, extending the know-how and conceiving new architectures for the joint allocation of heterogeneous resources is the next logical step and addresses uses cases that require the allocation of both computing and storage resources.

To achieve the goal of effective interoperability, two aspects are also expected to play key roles in addition to standardization: i) the definition of common, standard data models and ii) the use of open source software, offering common core components and allowing "plug-ins" for different applications and vendor devices. Although some vendors may still include proprietary optimizations, a common basis is expected to improve interoperability performance.

Ongoing efforts at the SDOs regarding the definition of common information models (e.g. related to network topologies) are a step in this direction. Nevertheless, the goal of achieving total interoperability still remains a hard issue, even more difficult in the domain of optical transport networks.

\section{Conclusions}

A GMPLS/PCE control plane for flexi-grid networks orchestrated by the ANM requires an architecture and protocols fulfilling the initial requirements while ensuring robustness, security, and scalability. Although the framework is considered to be stable and quite mature, addressing the constraints associated to flexi-grid DWDM networks, variable bandwidth transceivers and programmable devices is a complex problem. We have detailed the components of such a multi-domain control plane. The summarization of TE capabilities per domain, underlay network abstraction and applicability of stateful PCE capabilities to end-to-end path computation across multi-domain networks are part of IDEALIST solutions based on a hierarchy of the PCE, which have been implemented, demonstrated in a multi-vendor testbed and reported for standardization.

While other standardization bodies are working on the specification of the architecture of an SDN-based solution for multi-domain transport networks, our original goal was to extend the GMPLS protocol suite. The final architecture shares several aspects with SDN: since each domain is scoped and encapsulated by an active stateful PCE, and architectural elements still apply even if the network is composed of heterogeneous control technologies, including, for example, SDN and Openflow [15].

The architecture is hybrid, combining distributed and centralized elements. An additional role of the ANM and PCE is to enable a progressive migration to a transport SDN, since the architecture fits in a wider SDN applicability context in which driving a GMPLS domain is one south-bound interface of an orchestrator. From the perspective of the ANM and the H-PCE, the main differences would be the mechanism to retrieve the topologies and the actual service provisioning, which would be either delegated (GMPLS) or using a dedicated protocol that directly configures the hardware (OpenFlow).

A standard control plane for multi-domain/multi-vendor flexi-grid network can only be realistically designed assuming a standard data plane to a level of detail that does not currently exist. Current data plane standards imply that flexible network media channels are unlikely except in specially designed subnetworks and, while allowing mixed rate signals on the same fiber, standardized multi-vendor interoperability is not, as of today, 


\section{VIII. Acknowledgments}

\section{References} February 2012. October 2004. RFC 7491, March 2015 November 2015.

covered. IDEALIST has addressed this by having (data and control plane) implementation agreements, but without further advances (including, e.g. S-BVTs) a control plane cannot fully exploit the theoretical advantages of flexi-grid in an interoperable scenario. Interoperability still remains a major issue unlikely to be solved in the short term. While there are drivers and incentives (e.g. from operators or service providers trying to drive down costs), there remains a huge pressure for vendors.

This paper was supported by the European Community's Seventh Framework Programme FP7/2007-2013 through the IDEALIST (Industry-Driven Elastic and Adaptive Lambda Infrastructure for Service and Transport Networks) project, grant agreement n. 317999. (http://www.ict-idealist.eu)

[1] ITU-T Recommendation G.872, Architecture of optical transport networks, October 2012

[2] ITU-T Recommendation G.694.1, Spectral grids for WDM applications: DWDM frequency grid,

[3] E. Mannie, ed., Generalized Multi-Protocol Label Switching (GMPLS) Architecture, IETF RFC 3945,

[4] E. Crabbe, I. Minei, S. Sivabalan, R. Varga, PCEP extensions for PCE-initiated LSP setup in a stateful PCE model,", IETF internet draft, work in progress, October 2015

[5] H. Gredler, J. Medved, S. Previdi, A. Farrel, S. Ray, "North-Bound Distribution of Link -State and TE Information using BGP", IETF internet draft, work in progress, October 2015.

[6] D. King and A. Farrel, A PCE-Based Architecture for Application-Based Network Operations, IETF

[7] D. King and A. Farrel, The Application of the Path Computation Element Architecture to the Determination of a Sequence of Domains in MPLS and GMPLS, IETF RFC 6805, November 2012.

[8] L. Velasco, D. King, O. Gerstel, R. Casellas, A. Castro, V. Lopez, In-operation Network Planning, IEEE Communications Magazine, Vol. 52, No. 1, pp. 52-60, January 2014.

[9] F. Paolucci, A. Castro, F. Fresi, M. Imran, A. Giorgetti, B. B. Bhownik, G. Berrettini, G. Meloni, F. Cugini, L. Velasco, L. Potì, P. Castoldi, Active PCE demonstration performing elastic operations and hitless defragmentation in flexible grid optical networks, Photonic Network Communications, Springer, vol. 29, issue 1, 2014, pp 57-66

[10] R. Munoz, R. Casellas, R. Vilalta, R. Martinez, Dynamic and Adaptive Control Plane Solutions for Flexi-Grid Optical Networks Based on Stateful PCE, in Lightwave Technology, Journal of , vol.32, no.16, pp.2703-2715, Aug.15, 2014.

[11] O. González de Dios, R. Casellas, editors Framework and Requirements for GMPLS-Based Control of Flexi-Grid Dense Wavelength Division Multiplexing (DWDM) Networks, IETF RFC 7698,

[12] O. González de Dios, R. Casellas, R. Morro, F. Paolucci, V. López, R. Martínez, R. Muñoz, R. Vilalta, P. Castoldi, First Multi-partner Demonstration of BGP-LS enabled Inter-domain EON control with H-PCE , in Proceedings of OFC2015, 22-26 March 2015, Los Angeles (USA).

[13] O. González de Dios, R. Casellas, R. Morro, F. Paolucci, V. López, R. Martínez, R. Muñoz, R. Vilalta, Multi-partner Demonstration of BGPLS enabled multi-domain EON control and instantiation with H-PCE , Journal of Optical Communications and Networking, Vol. 7, No. 11.

[14] O. González de Dios, et al, First Demonstration of Multi-vendor and Multi-domain EON with SBVT and Control Interoperability over Pan-European Testbed, 41st ECOC2015, PDP4.1, September 2015, Valencia (Spain).

[15] R. Casellas, et al. , "SDN orchestration of OpenFlow and GMPLS flexi-grid networks with a stateful hierarchical PCE [invited]", IEEE/OSA Journal of Optical Communications and Networking, Vol. 7, No.1, A106-A117, Jan 2015 


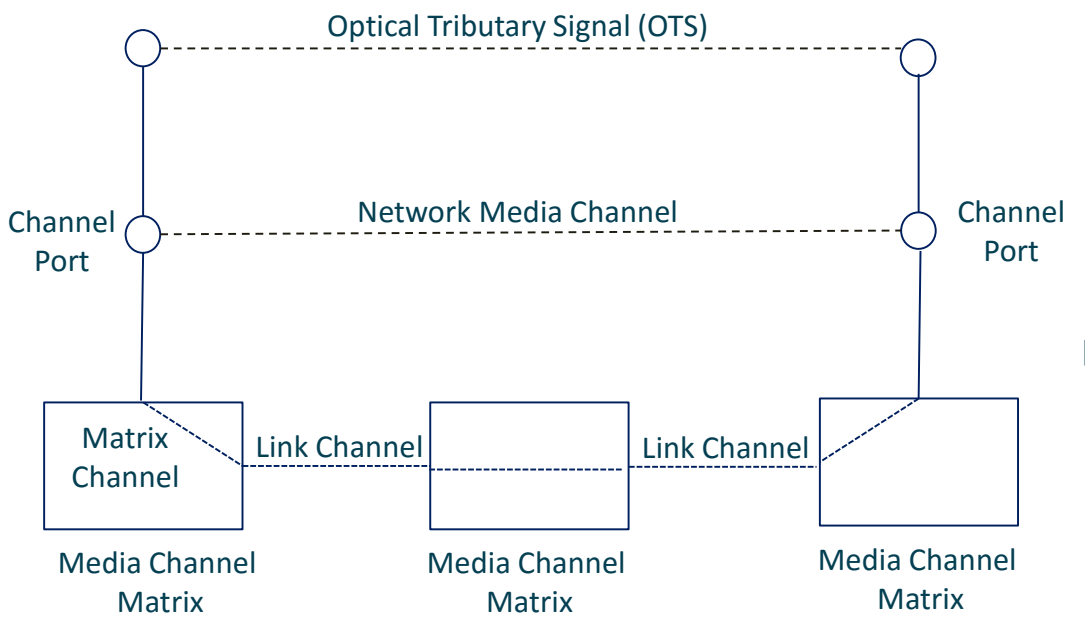

Network Media Channel

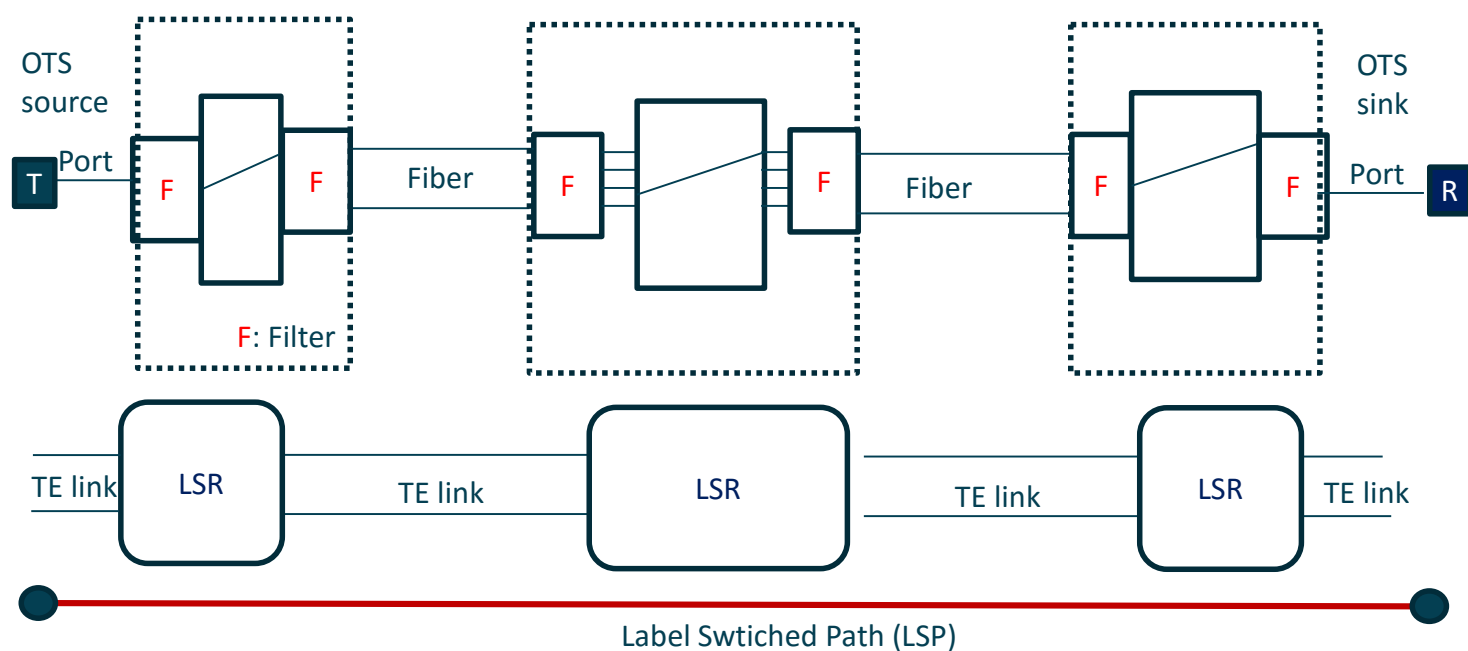

Figure 1 Relationship between Optical Tributary Signal, Network Media Channel and Media Layer Elements, and its view as a GMPLS LSP construct 


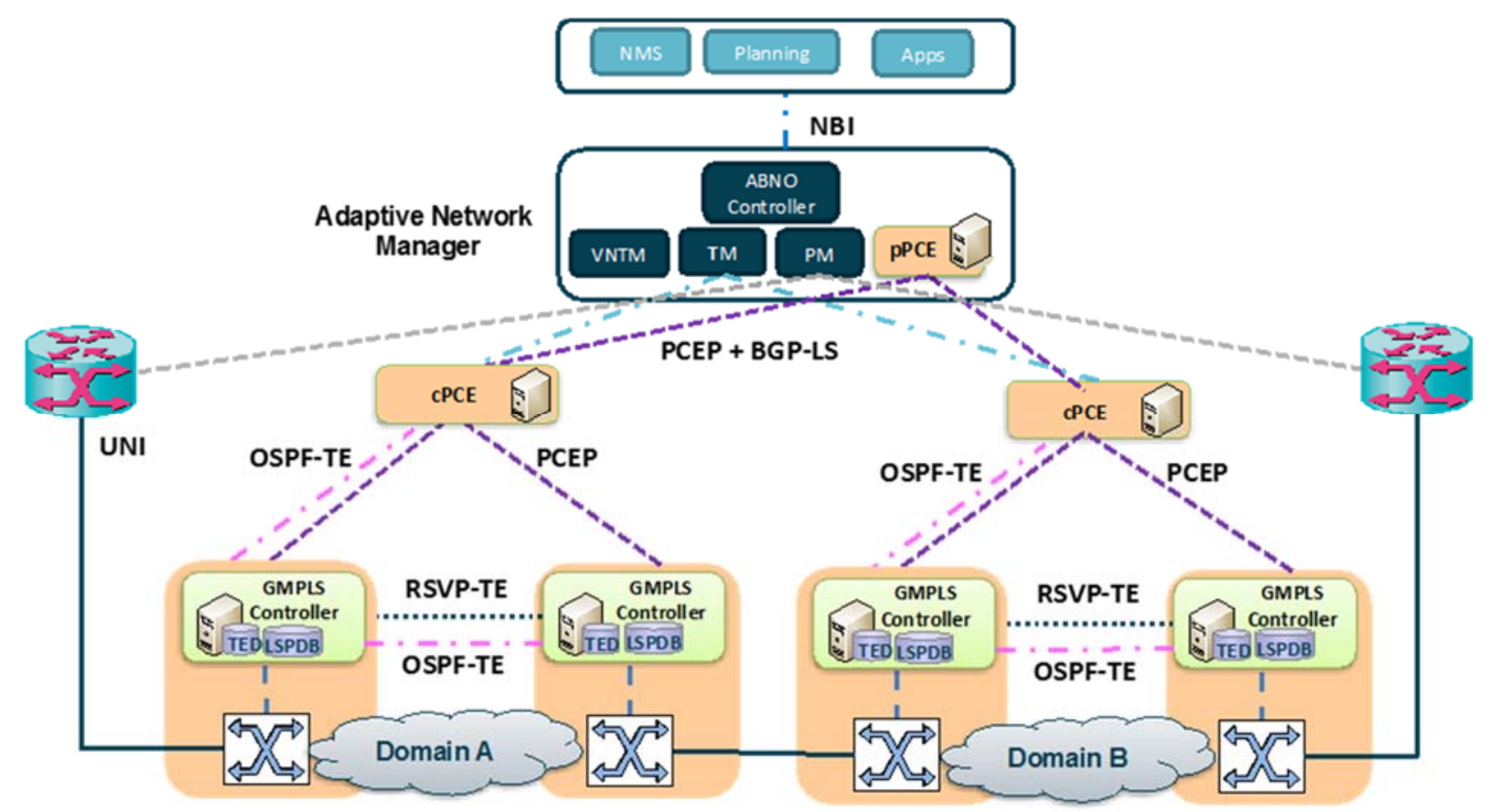

2 Figure 2 Control Plane architecture showing a multi-domain network with an AS-PCE per domain acting as 3 a Child PCE, a Parent PCE and an ANM. 


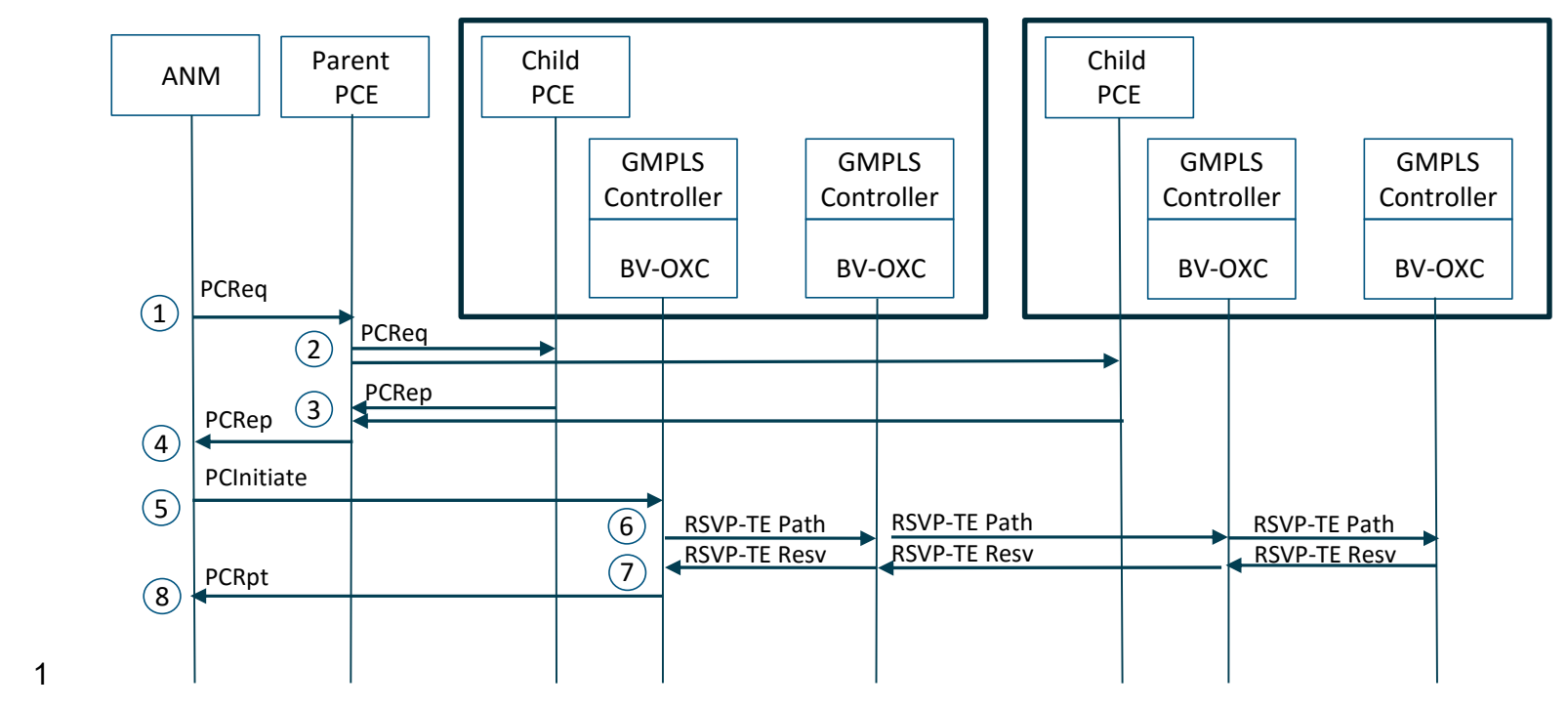

2 Figure 3 Single Session provisioning model, with stateless H-PCE 


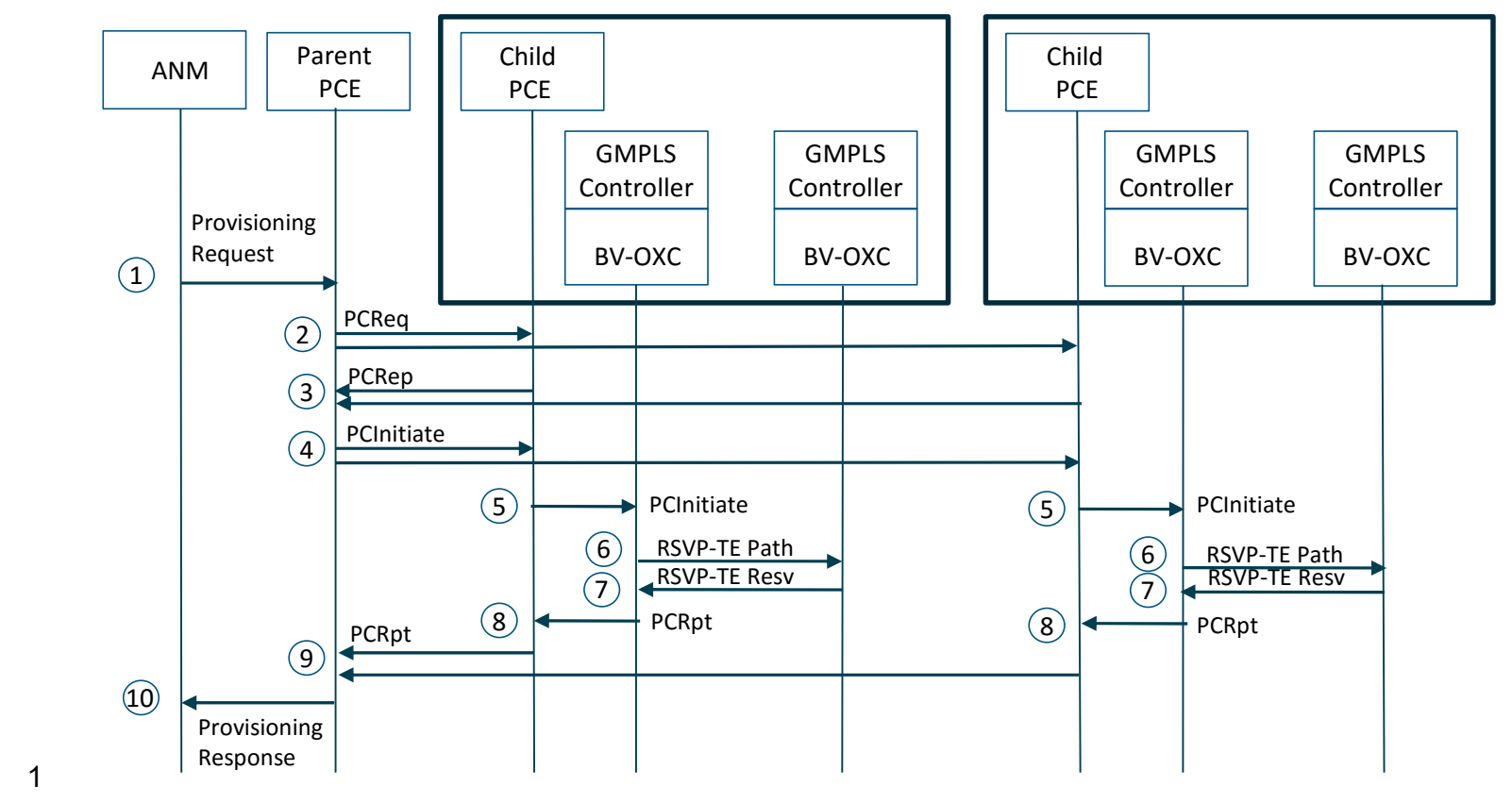

2 Figure 4 Stateful H-PCE with per-domain instantiation and local RSVP-TE session provisioning model 


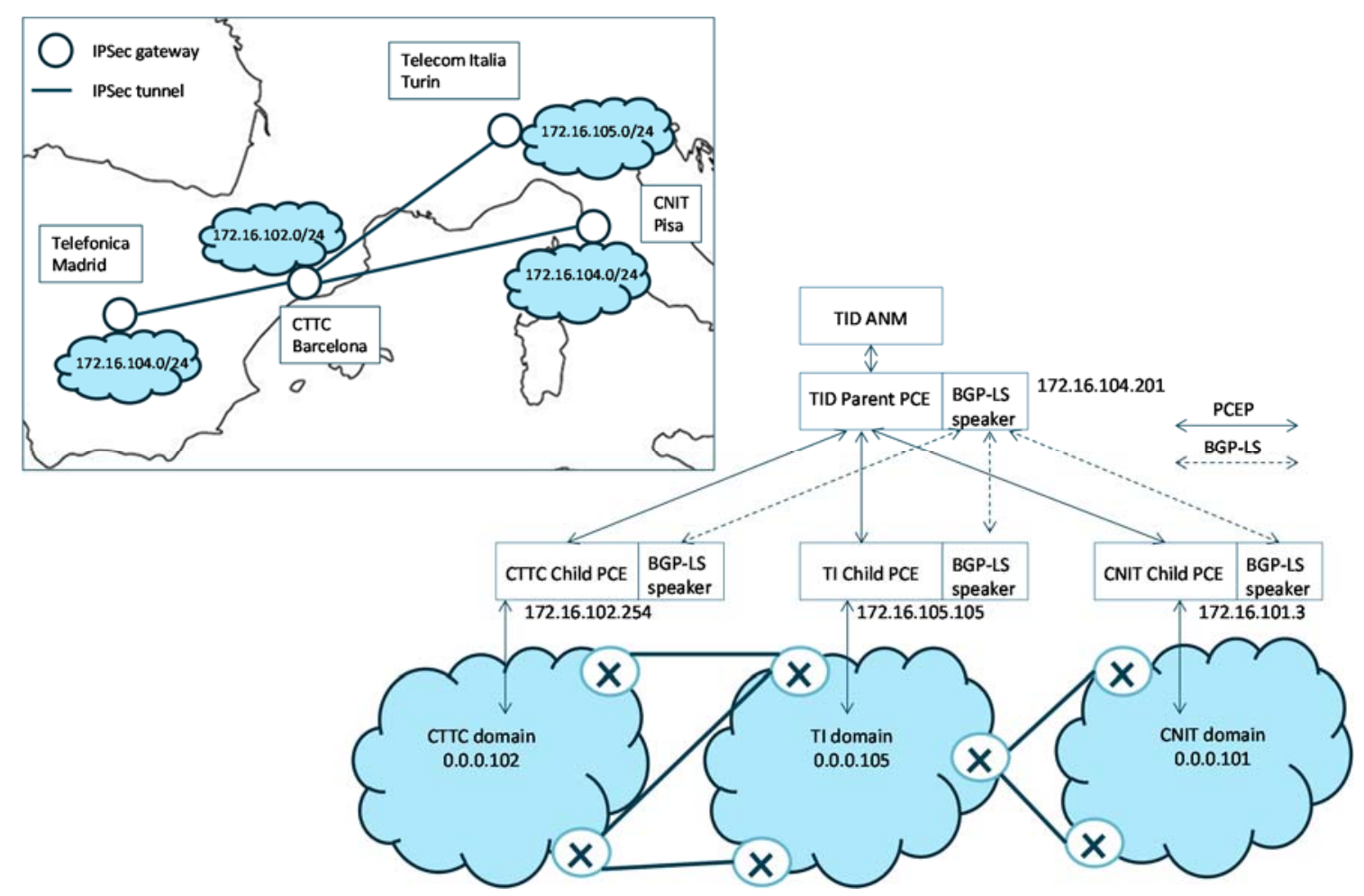

2 Figure 5 Multi-domain flexi-grid elastic optical network resulting from interconnecting partners' testbeds. 